CARDIOVASCULAR MEDICINE

\title{
Association of increased carotid intima-media thickness with the extent of coronary artery disease
}

\author{
A Kablak-Ziembicka, W Tracz, T Przewlocki, P Pieniazek, A Sokolowski, M Konieczynska
}

Heart 2004;90:1286-1290. doi: 10.1136/hrt.2003.025080

See end of article for authors' affiliations a thers affiliations

Correspondence to: Dr A Kablak-Ziembicka, Department of Cardiac and Vascular Diseases, Collegium Medicum Jagiellonian University, The John Paul II Hospital, Pradnicka 80, 31-202 Krakow, Poland; kablakziembicka@op.pl

Accepted 5 March 2004
Objective: To investigate (a) the relation between intima-media thickness (IMT) in carotid arteries and the extent of coronary artery disease (CAD); and (b) whether IMT is predictive of coronary atherosclerosis. The coexistence of severe extracranial atherosclerosis in patients with CAD was also analysed.

Methods: Coronary angiography and carotid ultrasound evaluation were performed in 558 consecutive patients (438 men), with a mean (SD) age of 58.8 (9.2) years and suspected CAD. Mean IMT was measured at both carotid arteries and expressed as the mean aggregate value. The relation between IMT and severity of CAD was determined.

Results: A significant correlation between mean IMT and advancing CAD ( $p<0.0001)$ was found. Four independent predictors of CAD were found in the discriminant analysis: age $(p=0.0193)$, hyperlipidaemia ( $p<0.0001)$, smoking ( $p=0.0032)$, and IMT $(p<0.0001)$. A significant increase in IMT was observed among patients with one, two, and three vessel CAD. A log normal distribution of IMT values showed that if mean IMT was over $1.15 \mathrm{~mm}$, patients had a $94 \%$ probability of having CAD, with sensitivity of $65 \%$ and specificity of $80 \%$ in the patients with a high risk of CAD. The number of critically stenosed extracranial arteries increased with advancing CAD. None of the patients with normal coronary arteries had severe stenosis of the extracranial arteries. Severe carotid, vertebral, or subclavian stenosis was found in $16.6 \%$ of patients with three vessel CAD.

Conclusions: IMT increases with advancing CAD, patients with mean IMT over $1.15 \mathrm{~mm}$ have a $94 \%$ likelihood of having CAD, and the coexistence of CAD with severe stenosis of aortic arch arteries is relatively high and was found in $16.6 \%$ of patients with three vessel CAD.
C oronary artery disease (CAD) remains the main cause of death in developed countries, despite several prevention programmes widely pursued across Europe, the United States, and other countries.

Since treadmill testing and transthoracic echocardiography can have limited specificity and sensitivity in diagnosing CAD, other methods are required. ${ }^{1}{ }^{2}$ This applies especially to patients with atypical angina or with typical angina accompanied by a negative treadmill test and echocardiography, at the same time reflecting an overall need for an introduction of new complementary diagnostic instruments. The development of Doppler ultrasound machines, advanced operating software, and high resolution transducers facilitates comprehensive analysis of the intima-media thickness (IMT) in the peripheral vessels - that is, the carotid and femoral arteries. Thickening of the intima-media is commonly recognised as the initial stage in the development of atherosclerosis.

Myocardial infarction or other acute coronary syndromes are the first symptom of CAD in more than $50 \%$ of all patients with cardiovascular disease. Thus, early detection of CAD may well prove to be instrumental in introducing effective treatment and may contribute to reducing mortality, for example, through plaque stabilisation and more aggressive control of atherosclerotic risk factors. ${ }^{3}{ }^{4}$

The impact of hypertension, diabetes mellitus, hyperlipidaemia, cigarette smoking, age, and sex on IMT has already been well established. ${ }^{5-10}$ However, the association between IMT and atherosclerosis of the coronary arteries requires further investigation. ${ }^{11-13}$

Furthermore, concomitant severe carotid and vertebral atherosclerosis may increase the risk of periprocedural stroke in patients undergoing coronary artery bypass grafting or percutaneous procedures for CAD. In these patients, Doppler ultrasound examination offers the potential for simultaneous intervention in both carotid and coronary arteries. ${ }^{14}$

The present study aimed at investigating whether an increase in IMT is related to the severity of CAD, as well as whether IMT may be instrumental in identifying patients with or without CAD. The frequency of concomitant severe carotid and vertebral artery atherosclerosis in patients with CAD was also investigated.

\section{PATIENTS AND METHODS}

We examined 558 consecutive patients (438 men, 120 women), with a mean (SD) age of 58.8 (9.2) years (range 40-81), admitted with suspected CAD from November 2000 to July 2001.

Coronary angiographies and ultrasound quantification of IMT in the carotid arteries were obtained for all patients.

High resolution B mode, colour Doppler, and pulse Doppler ultrasonography of both carotid arteries were performed with an ultrasound machine (ALOKA 5500 SSD; Aloka Co, Tokyo, Japan) equipped with a $7.5 \mathrm{MHz}$ linear array transducer. Patients were examined in the supine position with the head tilted backwards. After the carotid arteries were located by transverse scans the probe was rotated $90^{\circ}$ to obtain and record a longitudinal image of the anterior and posterior walls. The maximum IMT was measured at the near and far walls of the common carotid artery, the bifurcation, and the internal carotid arteries and was expressed as a mean aggregate value. The IMT was assessed as normal if it did not exceed $1 \mathrm{~mm}$. With regard to the incidence of plaque (defined as a focal thickening of the intima-media complex

Abbreviations: $C A D$, coronary artery disease; IMT, intima-media thickness 
greater than $1.3 \mathrm{~mm}$ ), its maximum diameter was assessed and included in further analysis.

Furthermore, the grade of stenosis in the carotid and vertebral arteries was assessed through the increase in the peak systolic and end diastolic velocities (according to the criteria of Hood $a \mathrm{al}^{15}$ ), with a view to establishing the frequency of concomitant $\mathrm{CAD}$ and severe carotid and vertebral atherosclerosis. The carotid and vertebral atherosclerosis was considered severe when the grade of stenosis was $\geqslant 70 \%$. When Doppler ultrasound indicated severe stenosis of the carotid or vertebral artery, the actual grade of stenosis was confirmed by standard angiography.

All scans were obtained by the same experienced sonographer, who had no prior knowledge of the patients' clinical and angiographic characteristics.

The changes in mean IMT were analysed for patients with angiographically normal coronary arteries, one vessel CAD, two vessel $\mathrm{CAD}$, and three vessel CAD.

Coronary angiography was performed in the catheterisation laboratory by means of COROSCOP (Siemens AG, Munich, Germany), equipped with Quantcor QCA version 2.0 quantitative coronary analysis software. All angiographies were performed by the Selindger technique through femoral artery access. Patients with at least one lesion $\geqslant 50 \%$ within the main branches of the coronary arteries were considered to have significant CAD. Patients with minimal atherosclerotic lesions $(<50 \%)$ in coronary arteries were not included.

\section{Statistical analysis}

Four groups of patients were considered in the analysis: patients without atherosclerotic lesions on coronary angiograms and patients with one, two, and three vessel CAD. The distribution of IMTs in these groups was evaluated according to IMT quartiles. To establish the independent value of IMT in predicting $\mathrm{CAD}$ we used multivariable discriminant analysis. The relation between mean IMT values and the severity of CAD was determined by one way analysis of variance. Furthermore, non-parametric estimation of carotid mean IMT distributions was assessed with simple histograms based on a theoretical log normal distribution of IMT values in the patients with $\mathrm{CAD}$ and the patients without CAD. The positive predictive values, sensitivity, and specificity were calculated to visualise the discriminating power of IMT.

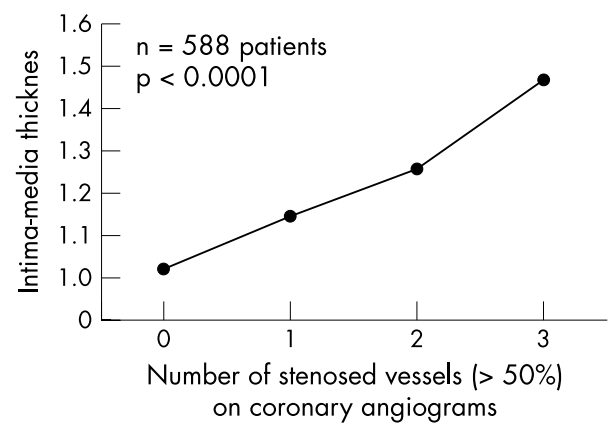

Figure 1 Relation between intima-media thickness (IMT) and advancing coronary artery disease (CAD) ( $p=0.0001$, analysis of variance) in patients with normal coronary arteries, with one vessel $C A D$, with two vessel $C A D$, and with three vessel $C A D$.

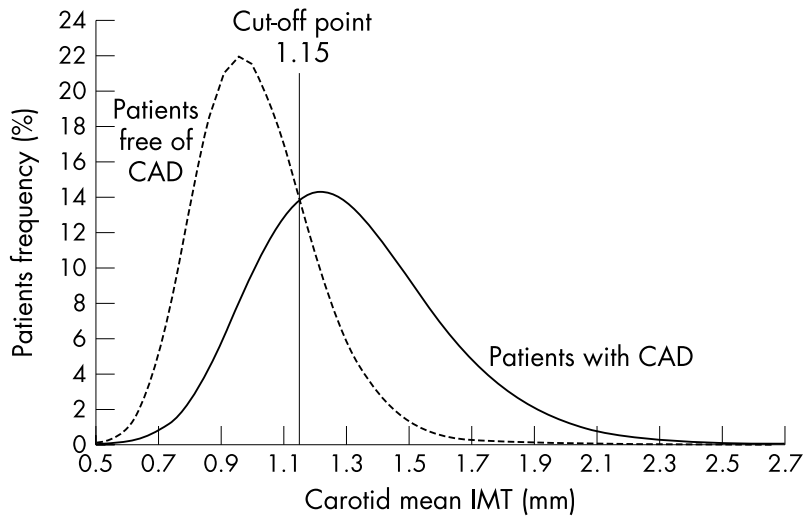

Figure 2 Theoretical log normal distribution of mean IMT in patients free of CAD and in patients with CAD. The cut off was set as the point where the curves cross.

Statistical analyses were performed with statistical software (Statistica 5.5, Stat Soft Inc, Tulsa, Oklahoma, USA). Values were considered significant if $\mathrm{p}<0.05$ (95\% confidence interval).

The frequency of concomitant CAD and severe carotid and vertebral stenosis was also calculated with one way analysis of variance.

Table 1 Clinical and angiographic characteristics of the studied patients

\begin{tabular}{llll}
\hline & $\begin{array}{l}\text { No CAD } \\
(\mathbf{n}=95)\end{array}$ & $\begin{array}{l}\text { CAD } \\
(\mathbf{n}=463)\end{array}$ & $\begin{array}{l}\text { p Value } \\
\left(\chi^{2}\right)\end{array}$ \\
\hline CCS class II, III, or IV & $81(85.3 \%)$ & $438(94.0 \%)$ & 0.0023 \\
ECG ischaemic changes & $22(23.2 \%)$ & $288(61.8 \%)$ & 0.0001 \\
Positive treadmill test & $58(61.0 \%)$ & $331 / 419(79.0 \%)$ & 0.0005 \\
Segmental contractility abnormalities on & $15(15.8 \%)$ & $328(70.4 \%)$ & 0.0001 \\
echocardiography & & & \\
Age (years) (mean) & $55.7(8.3)$ & $59.3(9.25)$ & 0.0001 \\
Men & $66(69.5 \%)$ & $372(80.3 \%)$ & 0.0188 \\
Obesity (BMI $\left.\geqslant 30 \mathrm{~kg} / \mathrm{m}^{2}\right)$ & $23(24.2 \%)$ & $94(20.3 \%)$ & 0.0859 \\
Hypertension & $53(55.8 \%)$ & $287(61.9 \%)$ & 0.5458 \\
NIDDM & $12(12.6 \%)$ & $91(19.7 \%)$ & 0.1755 \\
Cigarette smoking & $37(38.9 \%)$ & $298(64.4 \%)$ & 0.0001 \\
Hyperlipidaemia & $55(57.9 \%)$ & $390(84.2 \%)$ & 0.0001 \\
History of myocardial infarction & $12(12.6 \%)$ & $305(65.9 \%)$ & 0.0001 \\
Coronary angiography & $95(100 \%)$ & NA & NA \\
$\quad$ Normal coronary arteries & NA & $154(33.2 \%)$ & NA \\
One vessel CAD & NA & $104(22.5 \%)$ & NA \\
Two vessel CAD & NA & $205(44.3 \%)$ & NA \\
Three vessel CAD & \multicolumn{2}{|l}{} \\
\hline BMI, body mass index; CAD, coronary artery disease; CCS, Canadian Cardiovascular Society; NA, not \\
applicable; NIDDM, non-insulin dependent diabetes mellitus.
\end{tabular}




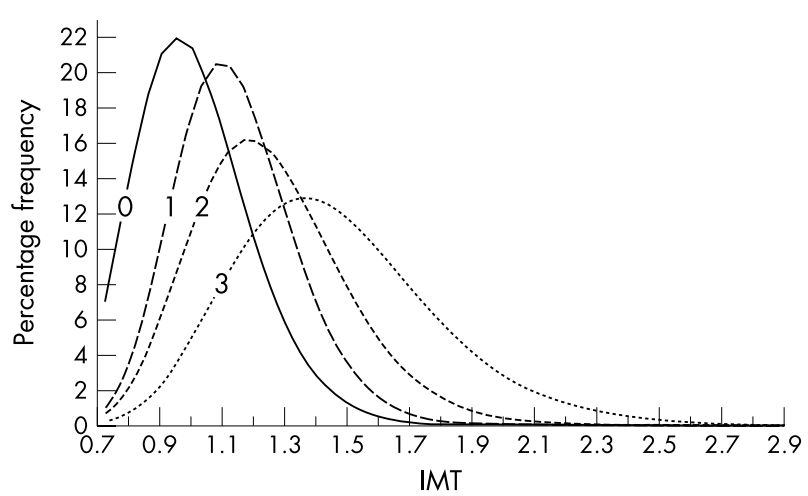

Figure 3 Theoretical log normal distribution of IMT in patients with normal coronary arteries and with one, two, and three vessel CAD. The cut off points were not calculated because of the overlapping distributions of IMT.

\section{RESULTS}

On coronary angiography $95(17.0 \%)$ patients had normal coronary arteries. One vessel CAD was diagnosed in 154 $(27.6 \%)$, two vessel CAD in $104(18.7 \%)$, and three vessel CAD in $205(36.7 \%)$ patients.

Table 1 shows selected characteristics of the study groups. We observed significant differences between the studied groups of patients without $\mathrm{CAD}$ and with $\mathrm{CAD}$ in the majority of examined factors. However, typical atherosclerosis risk factors and symptoms of CAD were relatively prevalent in patients without significant coronary lesions.

The mean measured IMT calculated for patients with normal coronary arteries was $1.01(0.19) \mathrm{mm}$, with one vessel CAD was $1.15(0.20) \mathrm{mm}$, with two vessel CAD was $1.26(0.27) \mathrm{mm}$, and with three vessel CAD was 1.47 (0.34) mm (fig 1). A significant, nearly linear correlation between IMT and advancing CAD ( $p<0.0001)$ was found. Patients with one, two, and three vessel CAD had significantly higher IMT than did patients without CAD $(\mathrm{p}=0.0044, \mathrm{p}<0.0001, \mathrm{p}<0.0001$, respectively $)$. We observed significant differences in IMT between patients with one and two vessel CAD $(p=0.0025)$, as well as between two and three vessel CAD $(p<0.0001)$.

Table 2 shows the distribution of patients according to IMT quartiles. Among patients without CAD 86\% had IMTs in the first and second quartiles $(<1.04 \mathrm{~mm}$ and $<1.2 \mathrm{~mm})$ and $81 \%$ with three vessel CAD had IMTs in the third and forth quartiles ( $\geqslant 1.2 \mathrm{~mm}$ and $\geqslant 1.4 \mathrm{~mm}$ ). Patients with more advanced $\mathrm{CAD}$ had a higher prevalence of intima-media complex thicknesses in the two upper quartiles. However, $2.1 \%$ of patients without significant CAD had IMTs in the third and forth quartiles, which is connected with the presence of plaque formation. Similarly, $4.4 \%$ of patients with three vessel CAD had IMTs in the lowest quartile.

To establish the independent value of IMT in predicting CAD we used discriminant analysis. To find how IMT behaves as a predictor of CAD severity in the presence of other important risk factor we included age, sex, hypertension, diabetes, hyperlipidaemia, cigarette smoking, and IMT in analysis. After a stepwise selection procedure we found four independent predictors of CAD in the final discrimination function: age $(\mathrm{p}=0.0193)$, hyperlipidaemia $(\mathrm{p}<$ $0.0001)$, smoking $(\mathrm{p}=0.0032)$, and IMT $(\mathrm{p}<0.0001)$.

We also created theoretical log normal distributions of IMT for patients with and without $\mathrm{CAD}$, first by drawing simple histograms, then by using them as the basis for drawing mean IMT distribution curves (fig 2). We took $1.15 \mathrm{~mm}$ as the cut off point. For that value we calculated the discriminating power of mean IMT measurements for

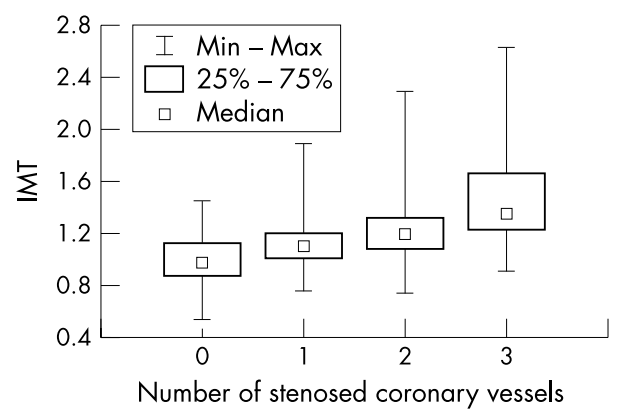

Figure 4 There is a large overlap between the groups with normal coronary arteries and with one, two, and three vessel CAD in the distribution of mean IMTs.

patients with and without CAD. Notably, the theoretical distributions of IMT showed that patients with a mean IMT over $1.15 \mathrm{~mm}$ had a $94 \%$ probability of having CAD, with a sensitivity of $65 \%$ and specificity of $80 \%$.

Furthermore, we sought the cut off points that would facilitate the allocation of patients into one, two, and three vessel CAD categories (fig 3). However, because of the overlapping distributions of IMT, this allocation procedure proved inadequate (fig 4).

We found that the number of critically stenosed carotid and vertebral arteries increased with advancing CAD (table 3). Among patients with normal coronary arteries none had severe stenosis of the aortic arch arteries, whereas we found severe stenosis of the internal carotid, vertebral, or subclavian artery in six $(3.8 \%)$ of the patients with one vessel CAD. Furthermore, we found a critical stenosis of these arteries in nine patients $(8.7 \%)$ with two vessel CAD and in 34 patients $(16.6 \%)$ with three vessel CAD (analysis of variance $\mathrm{p}=0.0001)$.

\section{DISCUSSION}

\section{Association between IMT of the carotid arteries and} prevalence of CAD

Our study group consisted of patients admitted to the department with suspected CAD. The majority of our patients had additional risk factors, a positive treadmill test, segmental hypokinesis or akinesis on echocardiography, ischaemic changes on ECG, or symptoms suggesting angina in functional class II or more. However, despite the high likelihood of CAD 17\% (95 patients) of these patients appeared not to have atherosclerotic lesions within their coronary arteries.

B mode ultrasonography of the carotid arteries, mainly thanks to the sufficient visualisation of carotid wall structures, offers the potential for effective evaluation of early atherosclerotic changes, such as thickening of the intima-media complex and non-significant carotid plaques, and brings into focus the possibility of monitoring atherosclerotic progress in peripheral arteries. ${ }^{16}$ This imaging technique may also hold some potential for predicting atherosclerotic lesions in vital arteries, such as coronary arteries or the aorta.

Many investigators have evaluated associations between the risk factors and mean IMT, as well as between the changes in risk factor levels and progression or regression in the thickness of the intima-media complex in the carotid arteries. $^{3-5}$ They found that the thickness of the intima-media complex increases with age, sex, hypertension, diabetes mellitus, hyperlipidaemia, and many other factors. ${ }^{5-10}$

Other studies compared IMT with the incidence of clinically symptomatic CAD and the extent or incidence of 
Table 2 Distribution of mean intima-media thickness (IMT) in 558 patients with and without CAD

\begin{tabular}{lclll}
\hline & Patients & $\begin{array}{l}\text { Lower quartile } \\
\text { (IMT <1.04) }\end{array}$ & $\begin{array}{l}\text { Second quartile } \\
(\mathbf{1 . 0 4} \leqslant \text { IMT }<1.2)\end{array}$ & $\begin{array}{l}\text { Third quartile } \\
(1.2 \leqslant \text { IMT }<1.4)\end{array}$ \\
\hline No coronary artery lesions & 95 & $55(57.9 \%)$ & $27(28.4 \%)$ & $11(11.6 \%)$ \\
One vessel CAD & 154 & $45(29.3 \%)$ & $63(40.9 \%)$ & $27(17.5 \%)$ \\
Two vessel CAD & 104 & $17(16.3 \%)$ & $29(27.9 \%)$ & $36(34.6 \%)$ \\
Three vessel CAD & 205 & $9(4.4 \%)$ & $30(14.6 \%)$ & $69(33.7 \%)$ \\
\hline
\end{tabular}

CAD on angiograms. ${ }^{12} 1718$ However, the results of these studies are rather discrepant.

In the present study we used mean aggregate IMT of the extracranial arteries (altogether 12 sites: two at each right and left common carotid artery, the bifurcation, and the internal carotid artery), including thickness of the plaque lesion, with a view to evaluating the association between the coronary artery status and the grade of carotid atherosclerosis. Mean aggregate IMT has been assessed in many previous studies, although in some of them it proved difficult to evaluate the internal carotid artery IMT precisely. ${ }^{19} 20$ However, technological advancement in ultrasound equipment and the availability of high resolution transducers has allowed for more accurate evaluation of arterial wall structures over the past decade.

The present study showed carotid artery IMT was higher in patients with angiographically confirmed CAD than in patients with normal coronary arteries. Thickening of the mean aggregate intima-media complex to just over $1.15 \mathrm{~mm}$ may prove to be predictive of significant CAD in nearly $95 \%$ of patients. Only a few patients with at least one vessel CAD have IMT within normal values. The number of patients with normal IMT decreases as the number of involved coronary arteries increases. However, in our study four of 205 patients $(1.9 \%)$ with three vessel CAD had an aggregate IMT below $1.0 \mathrm{~mm}$. However, both the sensitivity and specificity of IMT measurements are far from satisfactory.

Our observations are consistent with several previous studies. Crouse et $a l^{17}$ found a strong association between coronary artery status, verified by coronary angiography, and mean aggregate IMT in carotid arteries. Likewise, in our study mean aggregate IMT increased with advancing CAD.

Other investigators carried out population based studies in which IMT was evaluated with regard to the number and incidence of cardiovascular risk factors and acute coronary syndromes or stroke. ${ }^{21}$ A strong association between IMT and risk of stroke and myocardial infarction was established in the Rotterdam study..$^{22}$ Bots et $a^{21}$ used B mode ultrasonography to study the carotid arteries of 7983 patients aged 55 years and over. Throughout 4.6 years of observation (on average), they registered 194 new myocardial infarctions in the study group. Patients who had a myocardial infarction had significantly higher IMTs than did others. The statistical analysis confirmed a strong positive correlation between carotid IMT and the incidence of myocardial infarction, which prompted Bots et $a^{21}$ to conclude that IMT may effectively identify patients at high risk of myocardial infarction and cerebrovascular events.

Furthermore, O'Leary et al, ${ }^{23}$ having examined over 5800 patients ( $\geqslant 65$ years of age) with high resolution ultrasonography, found that increased IMT of the carotid arteries is directly associated with an increased risk of myocardial infarction and stroke in older adults without a history of cardiovascular disease. The relative risk of myocardial infarction, adjusted for age and sex, for the quintile with the highest thickness compared with the lower quintile was 3.87 and remained significant after further adjustment for traditional cardiovascular risk factors.

Since both the sensitivity and specificity of treadmill testing or echocardiography are limited, the introduction of IMT measurements of the carotid arteries may contribute significantly to diagnosing the status of patients with atypical chest pain or patients who are free of symptoms but with a number of cardiovascular risk factors. ${ }^{12}$ In these patients a high aggregated IMT in the carotid arteries may be a decisive factor in recommending coronary angiography and may warrant more aggressive risk factor control through the introduction of effective medical treatment such as statins or antihypertensive drugs.

Moreover, in our study a multivariable discrimination analysis determined that there are four independent predictors of CAD presence: age, hyperlipidaemia, smoking, and IMT. Thus, IMT may be considered another risk factor for $\mathrm{CAD}$, in the same way as it already is considered for hypertension according to the recommendations of the European Society of Cardiology published in 2003.

The results of our study indicate that measurement of IMT as a factor in gauging risk may have a certain potential for the preliminary identification of patients with or without CAD. We found IMT measurements to be highly instrumental in screening patients with suspected CAD but no history of myocardial infarction. Since over $50 \%$ of acute coronary syndromes (including myocardial infarction with ST segment elevation) are not accompanied by prodromal symptoms, we strongly believe that B mode quantification of carotid IMT can effectively identify patients at high cardiovascular risk. Through the equal distribution of the common atherosclerotic risk factors in studied groups (including hypertension, diabetes, and obesity), the impact of these risk factors on IMT in patients with normal coronary arteries and one, two, and three vessel CAD was diminished. However, more prevalent hyperlipidaemia, smoking habit, and higher age in patients

Table 3 Coronary artery status and severe stenosis ( $\geqslant 70 \%)$

\begin{tabular}{lclllc}
\hline $\begin{array}{l}\text { No of stenosed coronary } \\
\text { arteries }(\geqslant 50 \%)\end{array}$ & Patients & $\begin{array}{l}\text { Patients with } \\
\text { stenosis }\end{array}$ & $\begin{array}{l}\text { Stenoses of } \\
\text { SA }(\geqslant \mathbf{7 0 \%})\end{array}$ & $\begin{array}{l}\text { Stenoses of } \\
\text { VA }(\geqslant \mathbf{7 0 \%})\end{array}$ & $\begin{array}{c}\text { Stenoses of } \\
\text { ICA ( } \mathbf{7 0} \%)\end{array}$ \\
\hline 0 & 95 & $0(0 \%)$ & 0 & 0 & 0 \\
1 & 154 & $6(3.8 \%)$ & 4 & 1 & 2 \\
2 & 104 & $9(8.6 \%)$ & 1 & 3 & 6 \\
3 & 205 & $34(16.6 \%)$ & 2 & 3 & 39 \\
Total (patients with CAD) & 463 & $49(10.6 \%)$ & 7 & 7 & 47 \\
\hline
\end{tabular}

ICA, internal carotid artery; SA, subclavian artery; VA, vertebral artery. 
with more advanced CAD might have had some influence on IMT, but these factors are also associated with higher risk of atherosclerosis.

Regrettably, the extent of CAD cannot be predicted solely by the advancement of atherosclerosis in the carotid arteries. All attempts at allocating patients into groups with one, two, and three vessel CAD failed in our study as they did in the study of Adams et al. ${ }^{11}$ However, increasing mean aggregate IMT is strongly associated with more advanced CAD.

\section{The significance of severe carotid and vertebral stenosis in patients with CAD}

Our study indicates that advancing CAD is related to a gradually increasing rate of concomitant severe atherosclerosis in the extracranial arteries. Of 463 patients with at least one vessel CAD, $49(10.6 \%)$ had coexisting stenosis of the extracranial arteries of $\geqslant 70 \%$, whereas none of 95 patients without CAD had significant stenosis of the extracranial arteries. Furthermore, among patients with two and three vessel CAD, severe stenosis of the extracranial arteries was found in $8.6 \%$ and $16.6 \%$ of patients, respectively. Also, other investigators have found a high incidence of carotid and symptomatic coronary heart disease, estimated at 10-20\% according to various authors. ${ }^{24}$

It is our considered view that a relatively high coexistence of carotid and coronary atherosclerotic disease may justify Doppler ultrasound examination. Similarly, several investigators emphasise that patients with two and three vessel CAD should be considered for carotid atherosclerosis assessment, especially elderly men, smokers, patients with hypertension, or those with a high low density lipoprotein cholesterol concentration. ${ }^{25}$

Among patients referred for coronary artery bypass grafting the coexisting severe stenosis of carotid or vertebral arteries increases the periprocedural risk of stroke ${ }^{2627}$ Early diagnosis of concomitant carotid stenosis may affect the revascularisation strategy. Some patients may benefit from simultaneous coronary and carotid operations. ${ }^{14} 28$ Optionally, patients at high risk with advanced but stable CAD may require prior intervention in the carotid arteries followed by coronary intervention..$^{29-33}$

\section{Conclusions}

We found, firstly, that IMT increases with advancing CAD; secondly, that patients with a mean IMT over $1.15 \mathrm{~mm}$ have a high likelihood (94\%) of CAD (ultrasound examination of the carotid arteries can effectively predict atherosclerosis of the coronary arteries; and thirdly, that there is relatively high coexistence of CAD with severe aortic arch arteries stenosis. Ultrasound examination of the carotid and vertebral arteries should be considered for patients with advanced CAD who will be undergoing coronary artery bypass grafting.

\section{Authors' affiliations \\ A Kablak-Ziembicka, W Tracz, T Przewlocki, P Pieniazek, \\ M Konieczynska, Department of Cardiac and Vascular Diseases, The John Paul II Hospital, Krakow, Poland}

A Sokolowski, Krakow University of Economics, Krakow, Poland

\section{REFERENCES}

1 Hill J, Timmis A. Exercise tolerance testing. BMJ 2002;324:1084-7.

2 iliceto S, Galiuto L, Marangelli V, et al. Clinical use of stress echocardiography: factors affecting diagnostic accuracy. Eur Heart $J$ 1994; 15:672-80.

3 Crouse JR III, Byington RP, Bond MG, et al. Pravastatin, lipids, and atherosclerosis in the carotid arteries (PLAC II). Am J Cardiol 1995;75:455-9.
4 Byington RP, Miller ME, Herrington D, et al. Rationale, design, and baseline characteristics of the prospective randomised evaluation of the vascular effects of norvasc trial (PREVENT). Am J Cardiol 1997;80:1087-90.

5 Cuspidi C, Ambrosioni E, Mancia G, et al. Role of echocardiography and carotid ultrasonography in stratifying risk in patients with essential hypertension: the assessment of prognostic risk observational survey $J$ Hypertens 2002;20:1307-14.

6 Davis PH, Dawson JD, Riley WA, et al. Carotid intimal-media thickness is related to cardiovascular risk factors measured from childhood through middle age. The muscatine study. Circulation 2001;104:2815-9.

7 Suarez C, Lara I, Blanco F, et al. Ultrasonographic carotid findings associated to obesity in an elderly patients. Am J Hypertens 2000;13:S205.

8 Sun Y, Lin CH, Lu CJ, et al. Carotid atherosclerosis, intima media thickness and risk factors: an analysis of 1781 asymptomatic subjects in Taiwan. Atherosclerosis 2002; 164:89-94.

9 Tanaka H, Dinenno FA, Monahan KD, et al. Carotid artery wall hypertrophy with age is related to local systolic blood pressure in healthy men. Arterioscler Thromb Vasc Biol 2001;21:82-7.

10 Zanchetti $A$, Bond MG, Hennig M. Risk factors associated with alterations in carotid intima-media thickness in hypertension: baseline data from the European lacidipine study on atherosclerosis. J Hypertens 1998;16:949-61

11 Adams MR, Nakagomi A, Keech A. Carotid intima-media thickness in only weakly correlated with the extent and severity of coronary artery disease. Circulation 1995:92:2127-34.

12 Craven TE, Ryu JE, Espeland MA. Evaluation of the association between carotid artery atherosclerosis and coronary artery stenosis. Circulation 1990;82:1230-42.

13 Held C, Hjemdahl P, Eriksson SV, et al. Prognostic implications of intimamedia thickness and plaques in the carotid and femoral arteries in patients with stable angina pectoris. Eur Heart J 2001;22:11-4.

14 Chang BB, Darling RC, Shah DM, et al. Carotid endarterectomy can be safely performed with acceptable mortality and morbidity in patients requiring coronary artery bypass grafts. Am J Surg 1994;168:94-6.

15 Hood DB, Mattos MA, Mansour A, et al. Prospective evaluation of new duplex criteria to identify $70 \%$ internal carotid stenosis. J Vasc Surg 1996;23:254-61

16 Riley WA. Carotid intima-media thickness: risk assessment and scanning protocol. Eur Heart J 2002;23:916-8.

17 Crouse JR, Craven TE, Hagaman AP, et al. Association of coronary disease with segment-specific intimal-media thickening of the extracranial carotid artery. Circulation 1995;92:1141-7.

18 Nagai Y, Metter J, Earley CJ, et al. Increased carotid intimal-media thickness in asymptomatic older subjects with exercise-induced myocardial ischemia. Circulation 1998;98:1504-9

19 Heiss G, Sharett AR, Barnes R, et al. Carotid atherosclerosis measured by B-mode ultrasound in populations: association with cardiovascular risk factors in the ARIC study. Am J Epidemiol 1991;134:250-6.

20 Howard G, Burke GL, Evans GW, et al. Relationship of intimal-media thickness among sites within the carotid artery as evaluated by B-mode ultrasound. Stroke 1994;25:1581-7.

21 Bots ML, Hoes AW, Koudstaal PJ, et al. Common carotid intima-media thickness and risk of stroke and myocardial infarction. The Rotterdam study. Circulation 1997;96:1432-7.

22 del Sol IA, Bots ML, Grobbee DE, et al. Carotid intima-media thickness at different sites: relation to incident myocardial infarction. The Rotterdam study. Eur Heart J 2002;23:934-40.

23 O'Leary DH, Polak JF, Kronmal RA, et al. Carotid-artery intima and media thickness as a risk factor for myocardial infarction and stroke in older adults. N Engl J Med 1999;340:14-22.

24 Al-Mubarak N, Roubin GS, Liu MW, et al. Early results of percutaneous intervention for severe coexisting carotid and coronary artery disease. J Am Coll Cardiol 1999;84:600-2.

25 Zheng $\mathbf{H}$, Yuqing $\mathrm{H}$, Lansheng $\mathrm{G}$, et al. Carotid atherosclerosis should be assessed in patients with coronary artery disease. Lancet 1999;353(suppl 3): 14

26 Barnes RW, Liebman PR, Marszalek PB, et al. The natural history of asymptomatic carotid disease in patients undergoing cardiovascular surgery. Surgery 1981;90:1075-83.

27 Hines GL, Scott WC, Kofsky E, et al. Prophylactic carotid endarterectomy in patients with high-grade carotid stenosis undergoing coronary bypass: does it decrease the incidence of perioperative stroke? Ann Vasc Surg 1998; 12:23-7.

28 Sundt TM, Meyer FB, Fode NC, et al. Risk factors and operative results. In: Meyer FB, ed. Sundt's occlusive cerebrovascular disease, 2nd edn. Philadelphia: WB Saunders, 1994:241-7.

29 Terramani $\Pi$, Rowe VL, Hood DB, et al. Combined carotid endarterectomy and coronary artery bypass grafting in asymptomatic carotid artery stenosis. Am J Surg 1998;64:993-7.

30 Babatasi G, Theron J, Massetti M, et al. Value of percutaneous carotid angioplasty before cardiac surgery. Ann Cardiol Angiol 1996:45:24-9.

31 Kablak-Ziembicka A, Hlawaty M, Sadowski J, et al. [Management of coexistent advanced coronary artery disease and internal carotid artery stenosis]. Przegl Lek 2002;59:554-6.

32 Shawl F, Kadro W, Domanski MJ, et al. Safety and efficacy of elective carotid artery stenting in high-risk patients. J Am Coll Cardiol 2000;35:1721-8.

33 Wholey MH, Wholey M, Mathias K, et al. Global experience in cervical carotid artery stent placement. Catheter Cardiovasc Interv 2000;50:160-7. 